\title{
OBSERVATIONS OF BL LACERTAE OBJECT 1803+784 FROM THE GEODETIC VLBI ARCHIVE OF THE WASHINGTON CORRELATOR
}

\author{
C. E. TAteyama ${ }^{1}$ \\ CRAAM-INPE, Universidade Presbiteriana Mackenzie, Rua da Consolação 896, 01302-000 São Paulo, SP, Brazil \\ K. A. Kingham \\ US Naval Observatory, Earth Orientation Department, 3450 Massachusetts Avenue, NW, Washington, DC 20392 \\ P. KAUFMAnN ${ }^{2}$ \\ CRAAM, Universidade Presbiteriana Mackenzie, Rua da Consolação 896, 01302-000 São Paulo, SP, Brazil
}

AND

A. M. P. DE LUCENA

ROEN, Rádio Observatório Espacial do Nordeste, CRAAM-INPE, Estrada do Fio 6000, Eusébio, Fortaleza, CE, Brazil Received 2001 May 29; accepted 2002 March 19

\begin{abstract}
We present 11 maps of BL Lacertae object 1803+784 derived from geodetic VLBI observations archived at the Washington Correlator. The observations were obtained during the years 1988-1999. In this period three superluminal components with an expansion rate of 0.084 mas $\mathrm{yr}^{-1}$ were identified. A remarkable feature of the VLBI images is that the superluminal components only become visible on the jet at a radius of $1 \mathrm{mas}$ from the core, which was interpreted as a result of a helically curved jet. This feature and further evidence of helical patterns found in published high dynamic range VLBI maps at parsec scale and kiloparsec scale enabled us to obtain a well-constrained helical jet. The overall radio morphology of $1803+784$ could be described by two dominant components given by a narrow helical jet extending east-west about $6 \mathrm{kpc}$ from the core (deprojected size of $30 \mathrm{kpc}$ ) and a much larger north-south component perpendicular to the helical jet (cone axis) with a linear size of about $270 \mathrm{kpc}$.
\end{abstract}

Subject headings: BL Lacertae objects: individual $(1803+784)$ — galaxies: jets — radio continuum: galaxies — techniques: high angular resolution techniques: interferometric

\section{INTRODUCTION}

BL Lacertae $1803+784$ is a compact flat-spectrum radio source with $z=0.68$ (Witzel et al. 1988; Lawrence et al. 1996). It is a highly variable source in the optical and radio regimes (Wagner \& Witzel 1995), with high polarization and the featureless spectrum of a BL Lac object; however, it also has a quasar-like broad-line feature (Biermann et al. 1981). The radio images of $1803+784$ (Antonucci et al. 1986; Strom \& Biermann 1991; Kollgaard et al. 1992; Britzen et al. 1999) reveal the morphology of a double source at different spatial scales developing into a sequence of self-similar structures, with structure to the west at parsec scales bending around to the south at kiloparsec scales.

In light of the high activity and the excess of predicted X-ray emission in 1803+784 (Witzel et al. 1988; Ghisellini et al. 1993; Lähteenmäki \& Valtaoja 1999), superluminal motion would be expected; nevertheless, until now there has been no convincing detection of it. The first paper on this topic is Witzel et al. (1988). Using a sequence of images derived from geodetic VLBI data at several epochs in the 1980s, $1803+784$ showed a core-jet structure with a jet component stationary at 1.4 mas from the core. However, the location of the stationary component, as shown in other VLBI images available in the literature, revealed significant discrepancies. While VLBI observations obtained by Pearson \& Readhead (1988) for epochs earlier than that of

\footnotetext{
${ }^{1}$ INPE, São José dos Campos, SP, Brazil.

2 Part-time researcher at CCS, UNICAMP, Campinas, SP, Brazil.
}

Witzel's geodetic VLBI observations showed the stationary component at 1.2 mas, those VLBI observations made by Gabuzda et al. (1992, 1994) and Gabuzda \& Cawthorne (1996) for epochs after Witzel's VLBI observations exhibited the same stationary component at 1.0 mas. BL Lac $1803+784$ appeared to be a source containing a bright core and a presumably stationary component that could be located between 1.0 and 1.4 mas.

The first report of superluminal motion on $1803+784$ was made by Krichbaum et al. (1993) in millimeter VLBI observations of components close to the core. These observations revealed a variable apparent velocity, $\beta_{\mathrm{app}}$, of $3 c-5 c$ for components interior to 1 mas. Gabuzda et al. (1994) measured an apparent velocity of $2.7 c$ for a short-lived component located at about 2 mas from the core. Britzen \& Krichbaum (1995) reported position shifts between 0.7 and 1.5 mas, with some components suggesting a $\beta_{\text {app }}$ as high as $9 c$. As in the case of the location of the stationary component, reports on the detection of superluminal components are inconsistent, which mostly reflects the complexity of the jet structure in $1803+784$.

In this work we present 11 images of $1803+784$ derived from geodetic VLBI observations at $8.3 \mathrm{GHz}$, obtained from the Washington Correlator's database covering $10 \mathrm{yr}$ of observation. The result shows three superluminal components with an expansion rate of 0.084 mas $r^{-1}$. The analysis of our VLBI data leads us to interpret the jet in terms of a helical structure. We discuss the radio morphology of $1803+784$ from parsec to kiloparsec scale. 
TABLE 1

8.4 GHz VLBI OBSERVATIONS OF $1803+784$

\begin{tabular}{|c|c|c|c|c|c|}
\hline $\begin{array}{l}\text { Epoch } \\
\text { (1) }\end{array}$ & $\begin{array}{l}\text { Name } \\
(2)\end{array}$ & $\begin{array}{l}\text { Antennas }{ }^{\mathrm{a}} \\
\text { (3) }\end{array}$ & $\begin{array}{l}\text { Peak Brightness } \\
\left(\mathrm{Jy} \mathrm{beam}^{-1}\right) \\
(4)\end{array}$ & $\begin{array}{l}\text { Synthesized Beam } \\
(\text { mas } \times \text { mas }) \\
(5)\end{array}$ & $\begin{array}{l}\text { Beam Angle } \\
\text { (deg) } \\
\text { (6) }\end{array}$ \\
\hline 1988 Oct 1 .......... & NAPS-E & EOvHrGbMV & 2.34 & $0.7 \times 0.6$ & -41 \\
\hline 1988 Oct $2 \ldots \ldots \ldots$ & PPM-N2 & AMHXJaU & 2.34 & $0.7 \times 0.6$ & -41 \\
\hline 1988 Oct $3 \ldots \ldots \ldots$ & PPM-S4 & ADsJaUC & 2.34 & $0.7 \times 0.6$ & -41 \\
\hline 1989 Dec $12 \ldots \ldots$ & POLAR-89 & VTEMQJ & 2.85 & $0.6 \times 0.5$ & -25 \\
\hline 1991 Dec $17 \ldots \ldots$. & GLOBAL & ABDJUSCEV & 1.24 & $0.6 \times 0.5$ & -59 \\
\hline 1992 Aug 3 ......... & IR752 & GMREV & 1.70 & $0.6 \times 0.5$ & 87 \\
\hline 1992 Aug 10 ....... & IR753 & GMREV & 1.70 & $0.6 \times 0.5$ & 87 \\
\hline 1993 Nov $9 . . . \ldots \ldots$ & NE28 & FAKWV & 1.32 & $0.6 \times 0.5$ & 40 \\
\hline 1994 Sep 8 ........... & NB16 & GYAIWTV & 1.52 & $0.8 \times 0.7$ & 28 \\
\hline 1995 Sep 19 ........ & NA125 & GFAKWNV & 1.82 & $0.6 \times 0.5$ & 79 \\
\hline 1996 Apr $24 \ldots \ldots$ & NXS10 & GAKNOV & 1.47 & $0.6 \times 0.6$ & -26 \\
\hline 1996 Sep $3 \ldots \ldots \ldots$ & NA175 & FAKNOV & 1.49 & $0.7 \times 0.6$ & -12 \\
\hline 1998 Oct $1 \ldots \ldots \ldots$ & RDV11 & AEBrLaMkSc & 1.65 & $1.4 \times 0.5$ & -53 \\
\hline 1999 Aug 17 ...... & NA329 & FAKNEV & 1.75 & $0.6 \times 0.5$ & -39 \\
\hline
\end{tabular}

a A = Gilcreek (Alaska, 26 m), Hr = HRAS85 (Texas, 26 m), C = SESHAN25 (China, $25 \mathrm{~m}$ ), Ds = DSS45 (Tidbinbilla, $34 \mathrm{~m}$ ), $\mathrm{E}=$ Westford (Massachusetts, $18 \mathrm{~m}), \mathrm{F}=$ Fortaleza (Brazil, $14 \mathrm{~m}$ ), $\mathrm{G}=$ Algopark (Ontario, $46 \mathrm{~m})$, $\mathrm{H}=$ HatCreek (California, $26 \mathrm{~m}$ ), I = Matera (Italy, $20 \mathrm{~m}$ ), B = Hartrao (South Africa, $26 \mathrm{~m}$ ), M = Mojave (California, $12 \mathrm{~m}$ ), $\mathrm{K}=$ Kokee (Hawaii, $20 \mathrm{~m}$ ), $\mathrm{L}=$ NL-VLBA (Iowa, $25 \mathrm{~m}$ ), D = Hobart26 (Tasmania, $26 \mathrm{~m}$ ), $\mathrm{N}=$ NRAO20 (Greenbank, $20 \mathrm{~m}$ ), Gb = NRAO140 (Greenbank, $43 \mathrm{~m}$ ), $\mathrm{O}=$ Ny Alesund (Norway, $20 \mathrm{~m}$ ), $\mathrm{P}=\mathrm{Plattvil}$ ( North Caroline, $5 \mathrm{~m}$ ), Ja = Kashima (Japan, $26 \mathrm{~m}$ ), $\mathrm{J}=$ Kashima (Japan, $34 \mathrm{~m}$ ), $\mathrm{R}=$ Richmond (Florida, $18 \mathrm{~m}$ ), $\mathrm{S}=$ Santiago12 (Chile, $12 \mathrm{~m}$ ), $\mathrm{T}=$ Onsala (Sweden, $20 \mathrm{~m}$ ), $\mathrm{U}=$ Kauai (Hawaii, $9 \mathrm{~m}$ ), $\mathrm{V}=$ Wettzell (Germany, 20 $\mathrm{m}$ ), $\mathrm{Ov}=$ OVRO130 (California, $40 \mathrm{~m}$ ), $\mathrm{Z}=$ VNDNBERG (California, $9 \mathrm{~m}$ ), Y = Ylow7296 (Yellowknife, $32 \mathrm{~m}$ ), $\mathrm{W}=$ NRAO85 (Green Bank, 26 m), Br = BR-VLBA (Brewster, $25 \mathrm{~m}$ ), La = LA-VLBA (Los Alamos, $25 \mathrm{~m}), \mathrm{Mk}=$ Mk-VLBA (Mauna Kea, 25 m), Sc = SC-VLBA (St. Crois, 25 m).

\section{OBSERVATIONS}

Data were obtained from geodetic dual-frequency VLBI experiments (Rogers et al. 1983) carried out by the Naval Observatory (Eubanks et al. 1991), the National Oceanographic and Atmospheric Administration (Carter, Robertson, \& MacKay 1985), the Crustal Dynamics Project, and the Space Geodesy Project (Coates et al. 1985; Smith \& Turcotte 1993). The VLBI observations were processed at the Washington VLBI Correlator at the US Naval Observatory (USNO).

All observations were obtained with Mark III dualfrequency VLBI receivers at both the $\mathrm{X}$ and the $\mathrm{S}$ bands (centered at 8.5 and $2.3 \mathrm{GHz}$, respectively), providing noise temperatures of 70-200 K. The X-band back end consists of eight individual channels of $2 \mathrm{MHz}$ bandwidth spanning the range 8.2-8.9 GHz. An exception was the experiment RDV11 in which only four channels were used, spanning the range 8.4-8.9 GHz. Table 1 lists all observations used in this work: column (1) gives the epochs of the observations, column (2) gives the names of the experiments, column (3) gives the antennas used in the experiments, column (4) gives the peak brightness of the images, column (5) gives the interferometric dirty beams, and column (6) gives the position angles (P.A.s) of the beams measured from north to east. We have used a restored circular beam of 0.6 mas for the images. The dynamic range, as given by the ratio of the peak flux per beam to the lowest positive contour in the images, is about $300: 1$, which restricts the image to a radius of about 2 mas. VLBI maps of high dynamic range obtained by Gabuzda et al. (1994), Ros et al. (1999), and Kellermann et al. (1998) show features as far away as 20 mas from the core. The data were calibrated and fringed using standard routines from the AIPS software package, and the images were produced using the self-calibration procedures (e.g., Pear- son \& Readhead 1984) of the Caltech Difmap software package.

Throughout this paper we will assume the standard Friedmann cosmology with $H_{0}=65 \mathrm{~km} \mathrm{~s}^{-1} \mathrm{Mpc}^{-1}$ and $q_{0}=0.5$. At the distance of $1803+784(z=0.68)$, an angular size of 1 mas corresponds to a linear distance of $6.1 \mathrm{pc}$.

\section{RESULTS AND DISCUSSION}

\subsection{Radio Images}

Figure 1 shows the VLBI images of $1803+784$, and Table 2 lists the model fitting parameters of the visibility data. The source exhibits the well-known core-jet structure with expansion occurring westward along the axis of right ascension. The jet component presents an elongated structure, which this work will explain as the result of closely spaced components along the jet axis. Figure 2 shows the sequence of maps plotted according to the epoch of observation to emphasize the evolution of moving components. A first examination of the maps in Figure 2 reveals that the jet component in the early epochs (Oct 88 and Dec 89 ) is constituted by a continuous extended structure along the jet. The centroid of the emission region is located at about 1.2 mas. During the later epochs, Nov 93, especially Sep 94 and Sep 95 , the jet component shows a structure of a single-peaked source at 1.5 mas. This change of the centroid of the source as a shift of main emission region is larger than error because of the visibility data or modeling the source. However, the structural change of the jet in the maps from Apr 96 to Aug 99 is more difficult to follow. Despite the perception of an expansion pattern in the source, as one can see by following the centroid of the jet component from the images in Figure 2, the expansion itself does not show a regular systematic shift in position with a gradual increase of the 

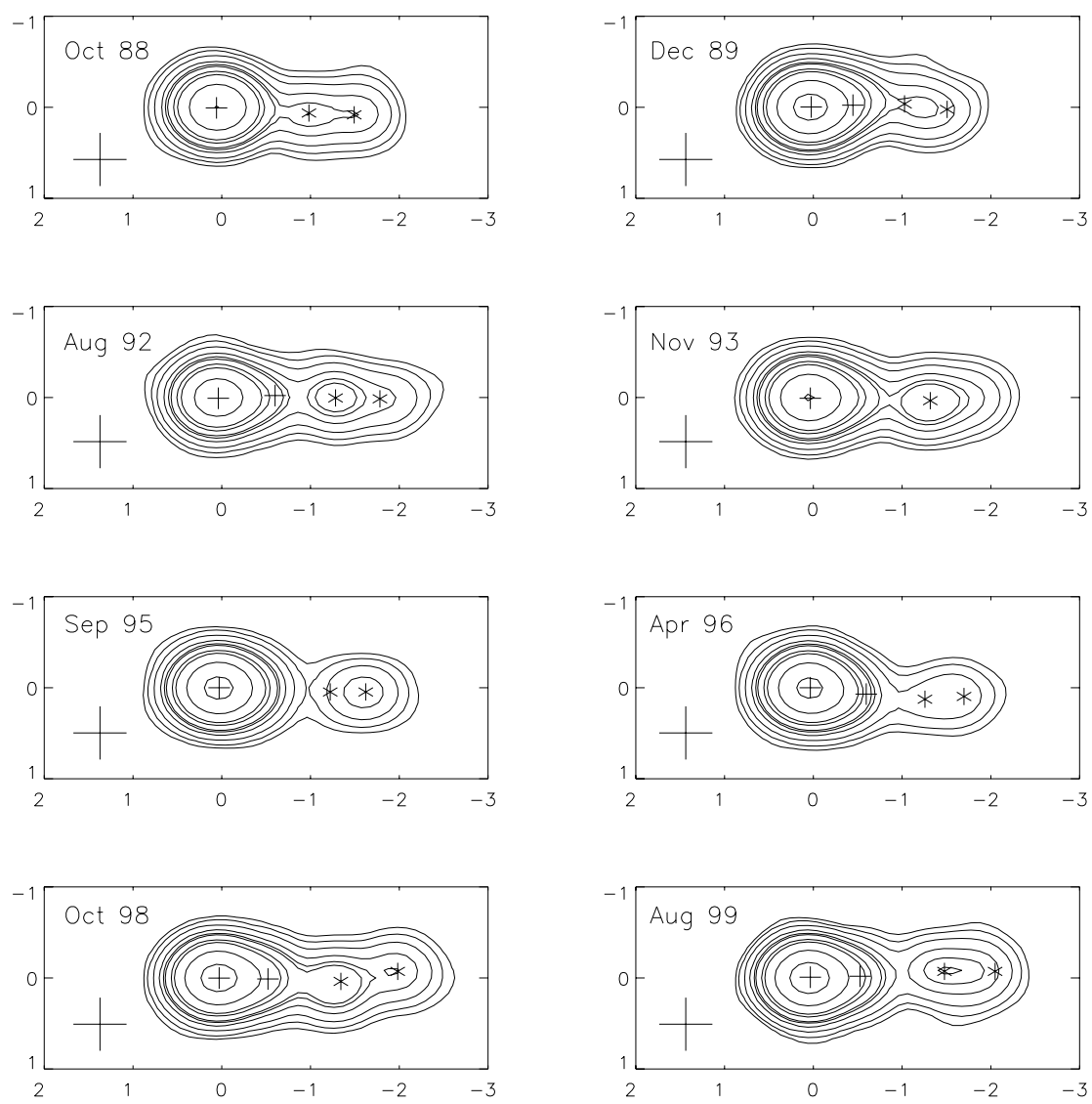
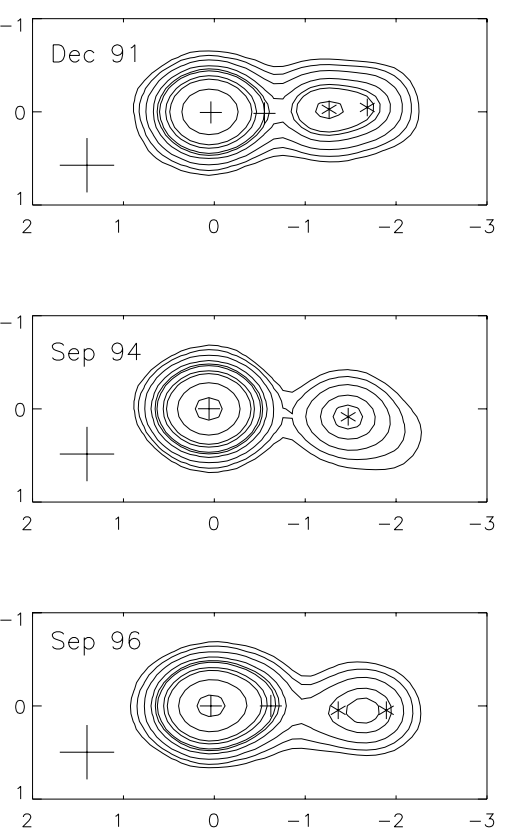

FIG. 1. $-8 \mathrm{GHz}$ VLBI images of $1803+784$. Contour levels are $1,2,4,8,12,15,24,32,64$, and 128 . The lowest contour level is $0.010 \mathrm{Jy}^{\mathrm{b}}$ beam ${ }^{-1}$, except for maps Oct 88, Dec 89, and Aug 92, which are $0.018 \mathrm{Jy}_{\text {beam }}{ }^{-1}$. The restored circular beam of 0.6 mas is represented by a cross on the bottom left of the maps. The axes are labeled in milliarcseconds. The stationary component (the VLBI core) is on the east side (left), while the moving component is on the west side (right). Model fits of the stationary components (core and stationary component at about 0.5 mas) are marked by crosses, and moving components are marked by asterisks.

moving component along the jet. A clue to decipher the jet behavior comes from the radio light curve. The high activity of the radio variability was taken as an indication that the extended jet structure could be a result of the superposition of multiple closely spaced components. Therefore, every time that a map (jet component) showed an elongated structure, we broke it into as many components as possible. A careful examination of the evolution of the components within the jet allowed us to derive an expansion pattern consistent with VLBI components of our maps.

\subsection{Model Fitting}

In order to decompose the jet into as many individual components as possible, the CLEAN image was fitted using Gaussian models of beam-convolved near point sources along the jet. In fact, the size of the components was arbitrarily set to 0.1 mas, with an axial ratio of 0.6 and major axis oriented to the jet axis (P.A. of $-90^{\circ}$ ). Most of the extended components were satisfactorily fitted using two Gaussian components. In a preliminary run, we note that the size of the core converged readily to a value of 0.2 mas for most images. The size of the core was then fixed to allow a better constraint on the fit of the remaining parameters of the source. We used the model fit routine in Difmap to fit the visibility data for each epoch. The simplest configuration has a core and a single point-source jet component (e.g., Sep
94), and the most complex configuration has an "extended core" component (core plus stationary component) and an extended jet component represented by two point sources. To line up four components in a long narrow core-jet structure is always critical, but the source has converged to a stable configuration. Although the expansion pattern described by the derived position components from map to map gave us the confidence that splitting the observed extended component into two point sources along the jet was correct, only observations with higher resolution would give a firm detection of individual components, especially in the maps from Apr 96 to Aug 99, where morphological structure appeared to be more complex.

For this purpose, we used the VSOP map of $1803+784$ obtained by Gabuzda (2000) at $5 \mathrm{GHz}$. The source was observed in 1998 July, which can be compared with our Oct 98 map and also our Aug 99 map. The VSOP map has a resolution of about 0.25 mas in the direction of the jet. We estimated the relative radial position of individual components to be $-0.4,0.0,0.9$, and 1.4 mas. Assuming that the easternmost component $(-0.4$ mas) is the core, the relative distance of VSOP components is in good agreement with our Gaussian positions. This gives a strong support to our derived components and the expansion pattern shown in Figure 2. The VSOP components are located along an arc, a change in P.A. of components that our data did not have the sensitivity to detect. This could affect the accuracy of the expan- 
TABLE 2

Model Fits to $1803+784$ at $8 \mathrm{GHz}$

\begin{tabular}{|c|c|c|c|c|c|}
\hline Epoch & Region & $\begin{array}{c}\text { Flux } \\
(\mathrm{Jy})\end{array}$ & $\begin{array}{c}r \\
\text { (mas) }\end{array}$ & $\begin{array}{l}\text { P.A. } \\
\text { (deg) }\end{array}$ & $\begin{array}{c}\theta^{\mathrm{b}} \\
(\mathrm{mas})\end{array}$ \\
\hline \multirow[t]{3}{*}{1988 Oct......... } & Core & 2.45 & 0 & 0 & 0.1 \\
\hline & $\mathrm{C} 1$ & 0.23 & 1.50 & -95 & 0.1 \\
\hline & $\mathrm{C} 2$ & 0.21 & 0.90 & -95 & 0.1 \\
\hline \multirow[t]{4}{*}{1989 Dec ........ } & Core & 2.77 & 0 & 0 & 0.1 \\
\hline & $\mathrm{C} 1$ & 0.22 & 1.51 & -94 & 0.1 \\
\hline & $\mathrm{C} 2$ & 0.20 & 1.09 & -90 & 0.3 \\
\hline & $\mathrm{E}$ & 0.65 & 0.40 & -81 & 0.2 \\
\hline \multirow{4}{*}{1991 Dec ........ } & Core & 1.38 & 0 & 0 & 0.1 \\
\hline & $\mathrm{C} 1$ & 0.14 & 1.71 & -90.9 & 0.1 \\
\hline & $\mathrm{C} 2$ & 0.27 & 1.28 & -87.4 & 0.1 \\
\hline & $\mathrm{E}$ & 0.06 & 0.58 & -85.1 & 0.1 \\
\hline \multirow[t]{4}{*}{1992 Aug........ } & Core & 1.68 & 0 & 0 & 0.2 \\
\hline & $\mathrm{C} 1$ & 0.11 & 1.80 & -90 & 0.1 \\
\hline & $\mathrm{C} 2$ & 0.40 & 1.27 & -95 & 0.1 \\
\hline & $\mathrm{E}$ & 0.32 & 0.48 & -93 & 0.01 \\
\hline \multirow[t]{2}{*}{1993 Nov ....... } & Core & 1.25 & 0 & 0 & 0.2 \\
\hline & $\mathrm{C} 2$ & 0.33 & 1.39 & -97.2 & 0.1 \\
\hline \multirow[t]{2}{*}{1994 Sep......... } & Core & 1.49 & 0 & 0 & 0.2 \\
\hline & $\mathrm{C} 2$ & 0.17 & 1.43 & -96.2 & 0.1 \\
\hline \multirow[t]{3}{*}{1995 Sep......... } & Core & 2.10 & 0 & 0 & 0.25 \\
\hline & $\mathrm{C} 2$ & 0.28 & 1.52 & -93.0 & 0.1 \\
\hline & $\mathrm{C} 3$ & 0.18 & 1.12 & -93.1 & 0.1 \\
\hline \multirow[t]{4}{*}{1996 Apr ....... } & Core & 1.57 & 0 & 0 & 0.2 \\
\hline & $\mathrm{C} 2$ & 0.10 & 1.59 & -91 & 0.1 \\
\hline & C3 & 0.04 & 1.10 & -97 & 0.1 \\
\hline & $\mathrm{E}$ & 0.18 & 0.44 & -101 & 0.1 \\
\hline \multirow[t]{4}{*}{1996 Sep......... } & Core & 1.56 & 0 & 0 & 0.2 \\
\hline & $\mathrm{C} 2$ & 0.15 & 1.73 & -92.4 & 0.1 \\
\hline & $\mathrm{C} 3$ & 0.10 & 1.25 & -96.1 & 0.1 \\
\hline & $\mathrm{E}$ & 0.17 & 0.51 & -89.2 & 0.1 \\
\hline \multirow[t]{4}{*}{1998 Oct......... } & Core & 1.60 & 0 & 0 & 0.2 \\
\hline & $\mathrm{C} 2$ & 0.13 & 1.82 & -97.0 & 0.1 \\
\hline & $\mathrm{C} 3$ & 0.26 & 1.30 & -93.7 & 0.1 \\
\hline & $\mathrm{E}$ & 0.41 & 0.40 & -93.3 & 0.1 \\
\hline \multirow[t]{4}{*}{1999 Aug........ } & Core & 1.89 & 0 & 0 & 0.2 \\
\hline & $\mathrm{C} 2$ & 0.09 & 1.86 & -90.0 & 0.1 \\
\hline & $\mathrm{C} 3$ & 0.19 & 1.33 & -88.1 & 0.1 \\
\hline & $\mathrm{E}$ & 0.28 & 0.62 & -77.8 & 0.1 \\
\hline
\end{tabular}

Note.-Axial ratio of the elliptical component $=0.6$; P.A. of the major axis of the elliptical component $=90^{\circ}$.

a P.A. of the component.

b Major axis of the elliptical component.

sion rate of the moving components but not the detection of superluminal components. Moreover, the arched structure of the VSOP map has provided an independent support to our helical model discussed in $\S 3.7$. All of the above resulted in a jet component dominated by the evolution of three individual components during the years 1988-1999. The superluminal components so detected were named $\mathrm{C} 1, \mathrm{C} 2$, and $\mathrm{C} 3$ and marked by asterisks on the images. The shift of component position from map to map is indicated by a diagonal line for each component in Figure 2.

\subsubsection{Moving Component}

The dominant component is $\mathrm{C} 2$, present in all images, and its position shifts from a radius of 0.9 mas in 1988 to 1.6 mas in 1999. C1 is an older component and is not seen in the images after 1992 August; C3 is a newer component that appears in our images in 1995 September.

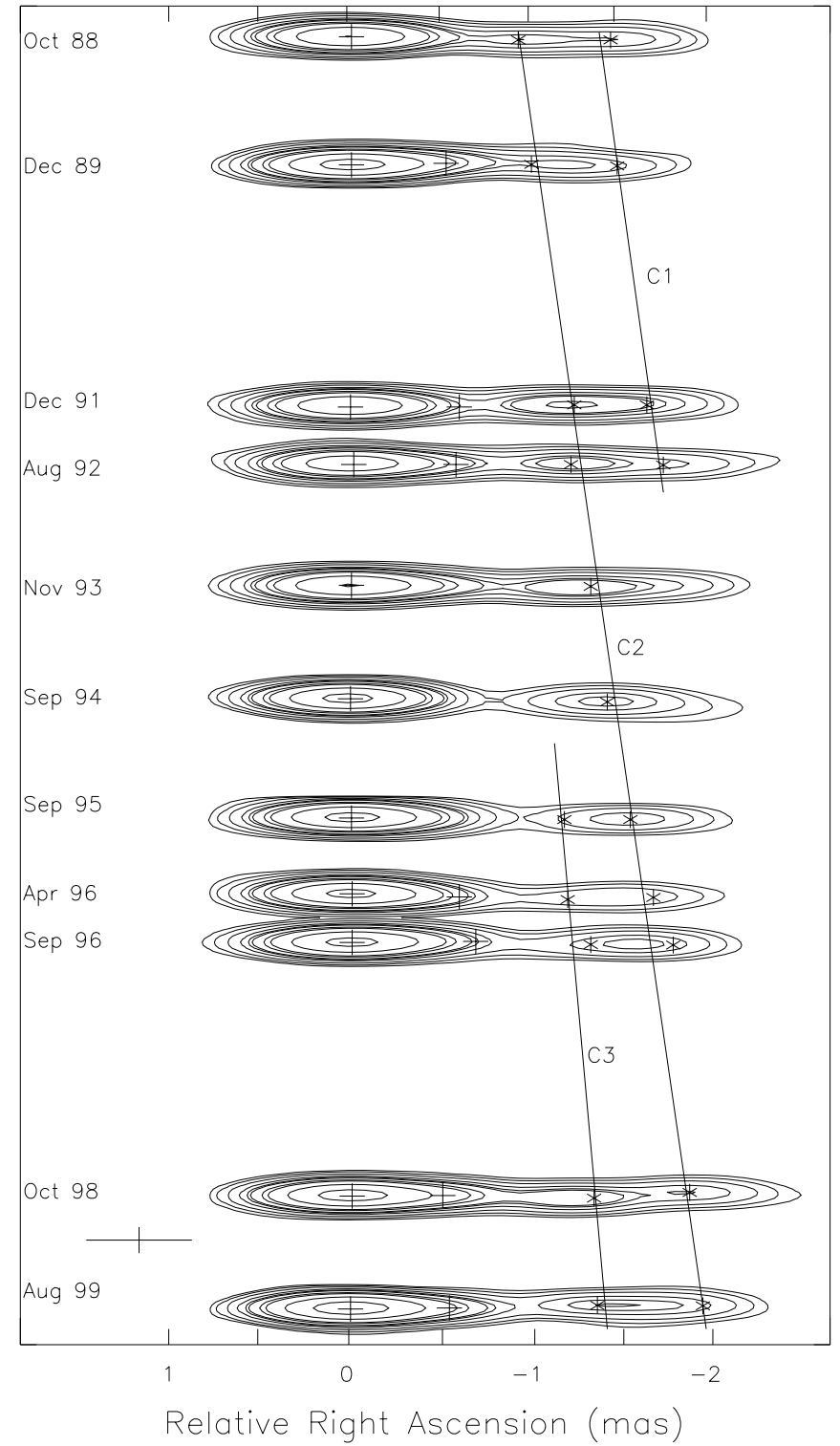

FIG. 2.-Time sequence of the maps emphasizing the evolution of moving components. The maps of Fig. 1 appear on this plot according to the epoch of observation along the vertical axis. The maps are stretched in right ascension (horizontal axis), and the scale is shown by a large cross corresponding to a circular beam of 0.6 mas on the bottom left of Fig. 2. The moving components, as indicated by diagonal lines, are labeled $\mathrm{C} 1, \mathrm{C} 2$, and $\mathrm{C} 3$.

\subsubsection{Stationary Component}

A weak stationary component is located at about 0.5 mas from the core, indicated by a cross on the images, and labeled as $\mathrm{E}$ in Table 2 . This stationary component appears in most of our maps ( 7 of 11). It has also been observed at the same position by Witzel et al. (1988) in VLBI observations made in 1985 at $22 \mathrm{GHz}$ and by Gabuzda et al. (1994) in 1990 at $5 \mathrm{GHz}$.

\subsubsection{Separation of Components with Time}

Figure 3 shows the separation of the components from the core versus time. The diagonal lines connecting the positions of components $\mathrm{C} 0, \mathrm{C} 1, \mathrm{C} 2$, and $\mathrm{C} 3$ represent an

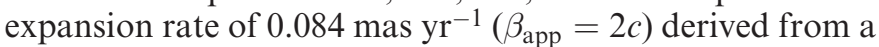




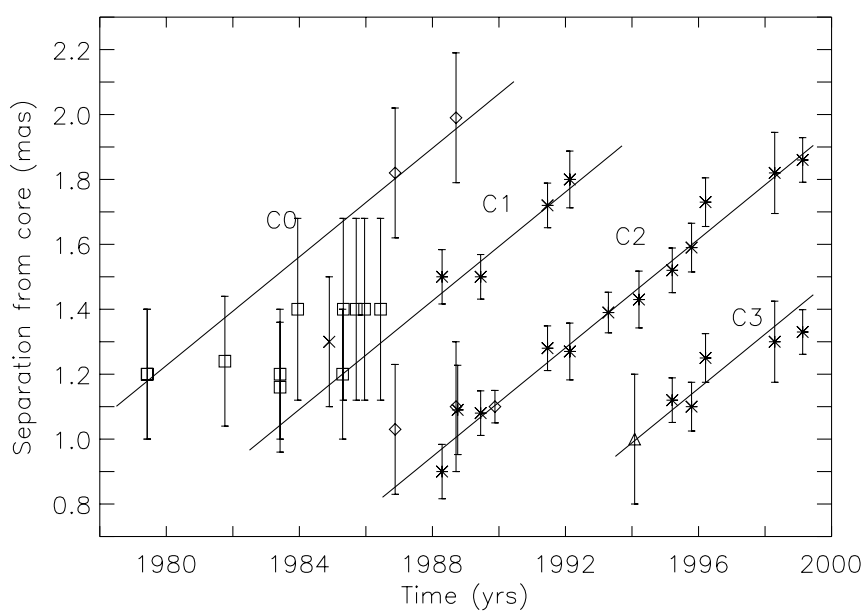

FIG. 3.-Component motions in $1803+784$. The vertical axis shows the separation in milliarcseconds of the component from the core. All data points before 1987 and represented by squares are from Witzel et al. (1988) and Pearson \& Readhead (1988), except one point (X) in 1985, which comes from Charlot (1990). Diamonds represent data from Gabuzda et al. (1992, 1994) and Gabuzda \& Cawthorne (1996), and the triangle in 1994 is from Fey et al. (1996). The diagonal lines correspond to the least-squares fit obtained from our main component $(\mathrm{C} 2)$. The error bars on component positions are assumed to be one-eighth of the beamwidth.

least-squares fit of the main component $\mathrm{C} 2$. Here, we have assumed that all components exhibit similar expansion rate. While $\mathrm{C} 1, \mathrm{C} 2$, and $\mathrm{C} 3$ are the moving components from our data (asterisks), $\mathrm{C} 0$ is an additional jet component based on VLBI observations made in two epochs by Gabuzda et al. (1994) at $5 \mathrm{GHz}$, which present a similar expansion rate $\left(0.08\right.$ mas yr $\left.^{-1}\right)$.

The component position at 1.0 mas from Gabuzda et al. $(1992,1994)$ and Gabuzda \& Cawthorne (1996), represented by a diamond on Figure 3, can be recognized as the first appearance of component $\mathrm{C} 2$ in the jet, and the point from the VLBA image (Fey, Clegg, \& Fomalont 1996), represented by a triangle, can be related to the first appearance of $\mathrm{C} 3$ in the jet. The 1.4 mas points from Witzel et al. (1988) and the 1.2 mas points from Pearson \& Readhead (1988) also appear consistent with the expansion pattern of VLBI components. A complex combination of evolution of different components within the jet, and the fact that the superluminal components only become visible on the jet at a separation of 1 mas from the core, made the detection of superluminal motion in $1803+784$ so elusive.

\subsection{Radio Variability}

Figure 4 shows the light curve of $1803+784$ at 5, 8, and 15 $\mathrm{GHz}^{3}$ As mentioned above, the radio variability strongly suggests a multicomponent source structure. Recent comparison between VLBI components and radio outbursts has revealed a complex relationship between these observables. While in sources such as BL Lac itself (Tateyama et al. 1998) and quasar 3C 345 (Valtaoja et al. 1999) the jet dominates the radio variability, in sources such as BL Lac OJ 287 (Tateyama et al. 1999) and quasar 3C 279 (Wehrle et al. 2001), the core is mostly responsible for the total radio flux

\footnotetext{
${ }^{3}$ Figure 4 data are from the University of Michigan Radio Astronomy Observatory, which is supported by the National Science Foundation and by funds from the University of Michigan.
}

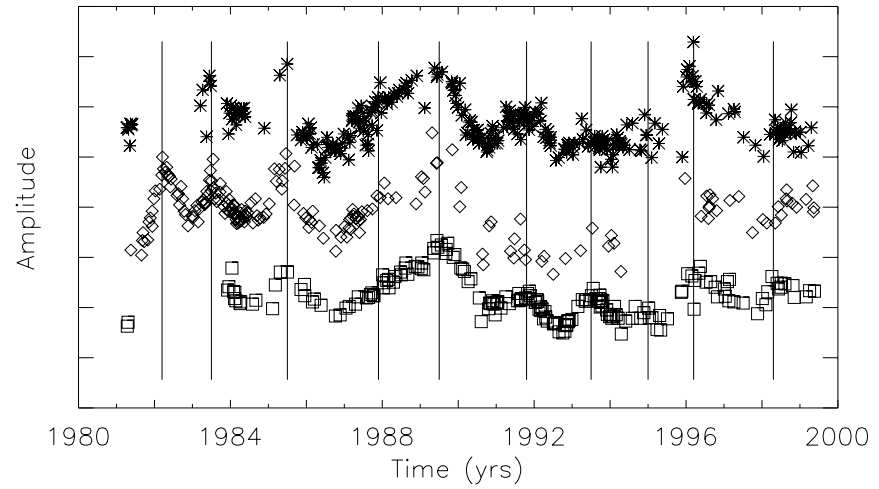

FIG. 4.-Radio light curves of $1803+784$. Squares correspond to 4.8 $\mathrm{GHz}$ data, diamonds correspond to $8.0 \mathrm{GHz}$ data, and asterisks correspond to $14.6 \mathrm{GHz}$ data. The vertical lines indicate individual outbursts.

variability. At the same time, in the case of OJ 287, the numbers of radio outbursts have been shown to be well correlated with the number of VLBI components. For simplicity, we have assumed here that the flux variability of the core is also connected with the change of the morphology of the jet. In Figure 4, vertical lines are used to indicate a structure that appears to be a single outburst. While outburst events occurring at about 1982.1, 1983.5, and 1985.5 show welldefined peaks, the events in 1988 and 1995 are not as easily seen, although some indication of an outburst is present in at least one of the radio light curves.

The radio light curves suggest an occurrence of one outburst approximately every $2 \mathrm{yr}$. Assuming that an outburst leads to the appearance of a new superluminal component that has an expansion rate of $\sim 0.1$ mas $^{-1} r^{-1}$, the separation between consecutive components would be about 0.2 mas or less. This means that 2-3 components would always be within the beam of our VLBI network. As can be seen from the light curves, the occurrence of outbursts is irregular, and possible fading or merging of individual components may occur in the inner jet before reaching the bend at 1 mas.

\subsection{The 1 mas Feature}

A remarkable feature of $1803+784$ is related to the lack of emission from the superluminal components between the core and the projected distance of 1 mas at centimeter wavelengths, a behavior shown by all moving components. We may consider three possible scenarios to interpret this feature:

1. A bent jet geometry with the outer jet axis oriented closer to the line of sight. In the inner segment, the jet components would move with a viewing angle nearly at the critical angle, a configuration that maximizes the apparent velocity of superluminal components but not the flux. The observed emission would come from the outer jet, which is oriented closer to the line of sight and more enhanced by Doppler boosting than the inner jet.

2. The emissivity of the jet components in the inner jet can be considered as an optically thick source at centimeter wavelengths until it becomes optically thin when the jet components reach a projected distance of 1 mas from the core (the outer jet). This scenario would account for the superluminal components detected close to the core in milli- 
meter VLBI observations made by Krichbaum et al. (1993) but not seen at centimeter wavelengths.

3 . The most likely scenario may be given by a combination of both, with a curved jet instead of a single bent jet and the spectrum of the source. Measurements of superluminal components from Krichbaum et al. (1993) have also shown a systematic variation of apparent velocities close to the core that indicate a helical structure. A relevant point related to our data is that the location of the lowest apparent velocity in their Figure 6, which would correspond in the helical framework to maximum Doppler factor, is coincident with the appearance of a weak stationary component at 0.5 mas in our images, suggesting that the superluminal components are detectable at $8 \mathrm{GHz}$ when passing this point. This also agrees with the fact that the stationary component is present in some of our maps and not in others, because its detection would depend on the superluminal components passing at the point of maximum Doppler boosting at the epoch of the VLBI observation or not. This reinforces the idea of multiple components, as suggested by the light curves of $1803+784$. After this point, the moving components would first depart from the line of sight and only become a strong radio source when the jets were pointing to Earth again. At this point, the superluminal component would be at about 1 mas from the core, with maximal Doppler boosting occurring at about 1.5 mas. Moreover, we would also have a relatively large loop so that the jet could be considered almost rectilinear, as seen in our VLBI maps.

\subsection{Helical Structure at Parsec (mas) Scale}

Inferences from observational aspects such as the stationary component at 0.5 mas from the core and the fact that the superluminal components appear only at a separation of 1 mas from the core led to the helical interpretation of our data. The high dynamic range images of Ros et al. (1999) at $8 \mathrm{GHz}$, observed at the end of 1991, and of Kellermann et al. (1998) at $15 \mathrm{GHz}$, observed at the beginning of 1997 , show remarkable bends or twists strongly supporting the helical jet. An examination of the maps suggests that the superluminal components follow the same curved path. For instance, the inner jet component (about 1 mas) on the image of Ros et al. (1999) can be seen to evolve westward and slowly bend toward the south to exhibit the jet structure (about 1.5 mas from the core) present on the image at 15 GHz. A well-defined helical curve in the maps can be described by an arc (concave down) centered in both images at about 2 mas from the core. We may then consider the stationary component at 0.5 mas from our data as a local maximum of Doppler boosting in the first loop, and the arc centered at about 2 mas from the core may correspond to the trajectory of a second loop. Farther out on the map at $15 \mathrm{GHz}$, an arc (concave up) centered about 4 mas from the core is another well-delineated helical feature. We estimate a helical amplitude to be about 0.5 mas at the center of the arc (4 mas from the core).

\subsection{Helical Structure at 100 mas and 2000 mas Scales}

Britzen et al. (1999) obtained VLBI maps showing structures at 100 and 2000 mas size scale at $1.66 \mathrm{GHz}$. The 100 mas scale map is a combination of VLBI and MERLIN data showing a double source structure with a bend to the north about 50 mas from the core. The 2000 mas scale map presents an extended westward structure along the axis of right ascension followed by elongated structure heading to the south showing a typical " dogleg" morphology (Stocke, Burns, \& Christiansen 1985). The bend toward the south is about 500 mas from the core and can be considered as a transition region from parsec to kiloparsec scale (Britzen et al. 1999). On the 100 mas scale map we take a peak located at $(-30,-5)$; hereafter, the numbers in the parentheses represent the relative right ascension and declination, respectively, in mas and a point located at $(-42,10)$ as reference points for helical modeling. The former is also well correlated with the outermost extended component on the maps of Ros et al. (1999) at 2.3 GHz.

\subsection{Helical Jet Model}

For modeling these features, we have considered the hydrodynamic helical jet model described by Hardee (1987). Application of this model has been made for BL Lac objects OJ 287 (Vicente, Charlot, \& Sol 1996) and BL Lac itself (Tateyama et al. 1998). A helical trajectory can be described by a moving feature on the surface of a cone of half-opening angle $\alpha$ and constant pitch angle $\Delta$, with the cone axis on the $z$-coordinate axis at angle $\chi$ to the observer's line of sight. For a constant isothermal jet expansion, the helical feature describes a spiral on the $(x, y)$ plane with amplitude

$$
A=A_{1}\left(\frac{z}{z_{1}}\right)^{d_{1}},
$$

and phase

$$
\phi=\phi_{1} \pm \ln \left(\frac{R}{R_{1}}\right) \frac{\tan \Delta}{\alpha},
$$

with

$$
\tan \Delta=\frac{2 \pi A_{1}}{\lambda_{1}},
$$

where $R=\Psi\left(z+z_{0}\right), \Psi$ is the half-opening angle of the jet, and $z_{0}$ is the origin of the cone; the values $A_{1}, \phi_{1}, \lambda_{1}$, and $R_{1}$ are the amplitude and phase of the helix, the helical wavelength, and radius of the jet, respectively, at distance $z_{1}$ from the origin; $d_{1}$ expresses the rate at which the helical amplitude grows, also evaluated at $z_{1}$. We have set $A=\xi R$, assuming that the ratio $\xi$ between the amplitude of the helix $A$ and the radius of the jet $R$ is constant; thus, $\alpha=\xi \Psi=\xi R_{1} /\left(z_{1}+z_{0}\right)$.

The helical features present on the map of Kellermann et al. (1998), Britzen et al. (1999), and our VLBI data provide a robust helical model with $\alpha=0.40, \Delta=2.36$, and $\chi=11^{\circ}$. The jet parameters are $\Psi=0.33, R_{1}=0.044$ mas, $\lambda_{1}=8.1 \mathrm{mas}, A_{1}=0.053 \mathrm{mas}, \phi_{1}=0.39, z_{0}=5.0 \mathrm{mas}$, $z_{1}=2.6 \mathrm{mas}, d_{1}=1.17$, and $\xi=1.2$.

Figure 5 shows two helical curves represented by solid and dashed lines. They are continuous lines describing helical structures from parsec to kiloparsec scale. The top panel shows the helical curve corresponding to parsec scale superposed on the sky position of components of our maps for all epochs. The large asterisk corresponds to an estimate of maximal helical amplitude on the image of Kellermann et al. (1998). This helical pattern can be compared with that derived by Krichbaum et al. (1993). A remarkable result of this helical model is that the inner helical curve nearest to the core can be compared in detail with the arched trajectory 

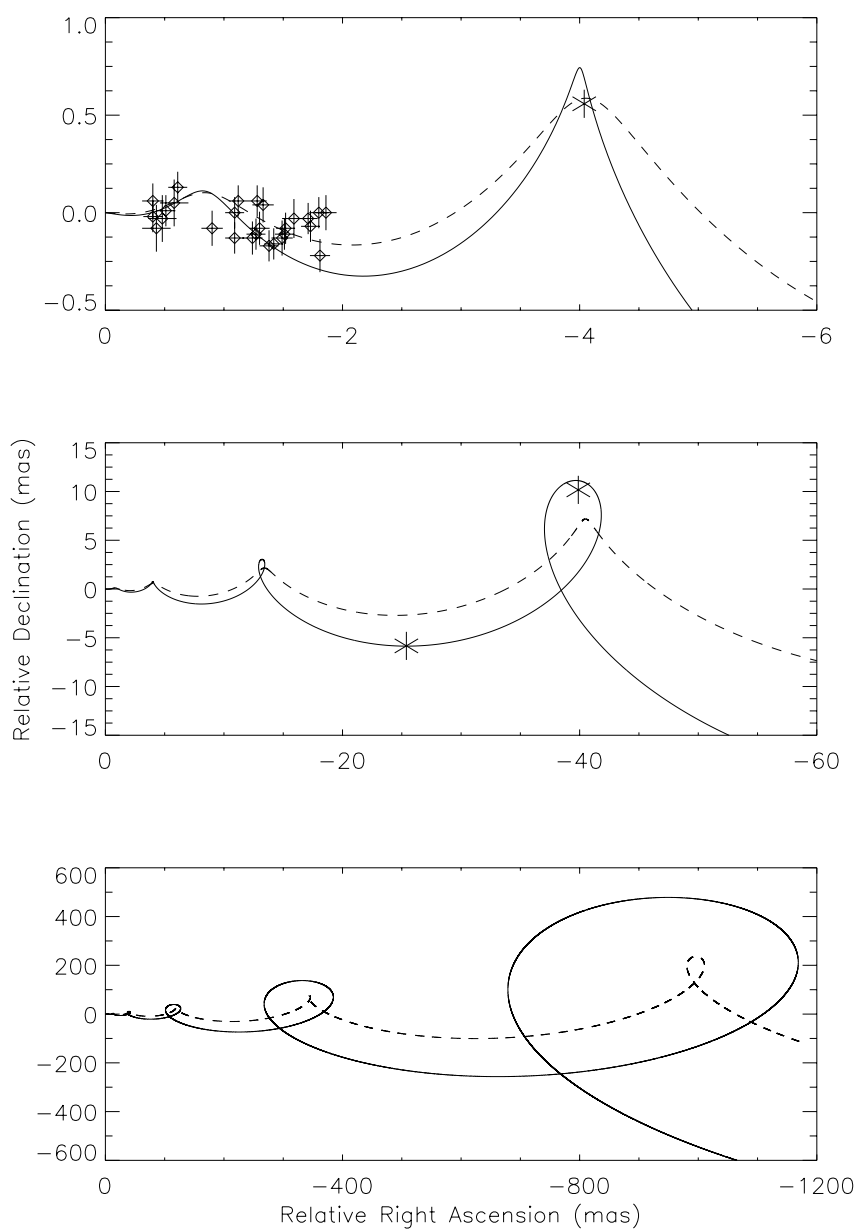

FIG. 5.-Helical model for $1803+784$. Sky positions are derived from the helical model. The solid line represents the best helical model, which accounts for radio features at parsec, intermediate (100 mas), and kiloparsec scales. The dashed line represents the same helical model modified so as to have the center of the loops at the same place but having smaller amplitudes to improve the fit at parsec scale (our VLBI data). The top panel shows the helical curves for parsec scale. Diamonds represent a superposition of sky positions of stationary component (at about 0.5 mas from the core) and the moving components in our maps for all epochs. The uncertainty is assumed to be one-eighth of the beamwidth. The large asterisk corresponds to the point on the center of the arc (maximal helical amplitude), as estimated from the image of Kellermann et al. (1998). The middle panel shows the helical curve at 100 mas scale. The large asterisks represent points of maximal amplitude along the helical trajectory on the map of Britzen et al. (1999). The bottom panel shows the helical curve at 2000 mas scale.

in which VSOP components (Gabuzda 2000) are distributed, reinforcing the helical model and providing further support that the easternmost VSOP component is the core. The middle panel shows the helical structure at 100 mas scale, and the large asterisks represent reference points of helical features on the map of Britzen et al. (1999). The bottom panel shows the helical structures at kiloparsec scale. Although the helical curve represented by the solid line gives only a reasonable fit to our data, it describes well all helical features present on the radio maps, from parsec to kiloparsec scale. The last loop in the 100 mas scale map (middle panel) agrees well with the lobe extending toward the north from the secondary component. Figure 6 shows the same helical curve superposed on the VLBI maps. Note that the lack of radio emission may suggest that Doppler boosting is still present on the jet.

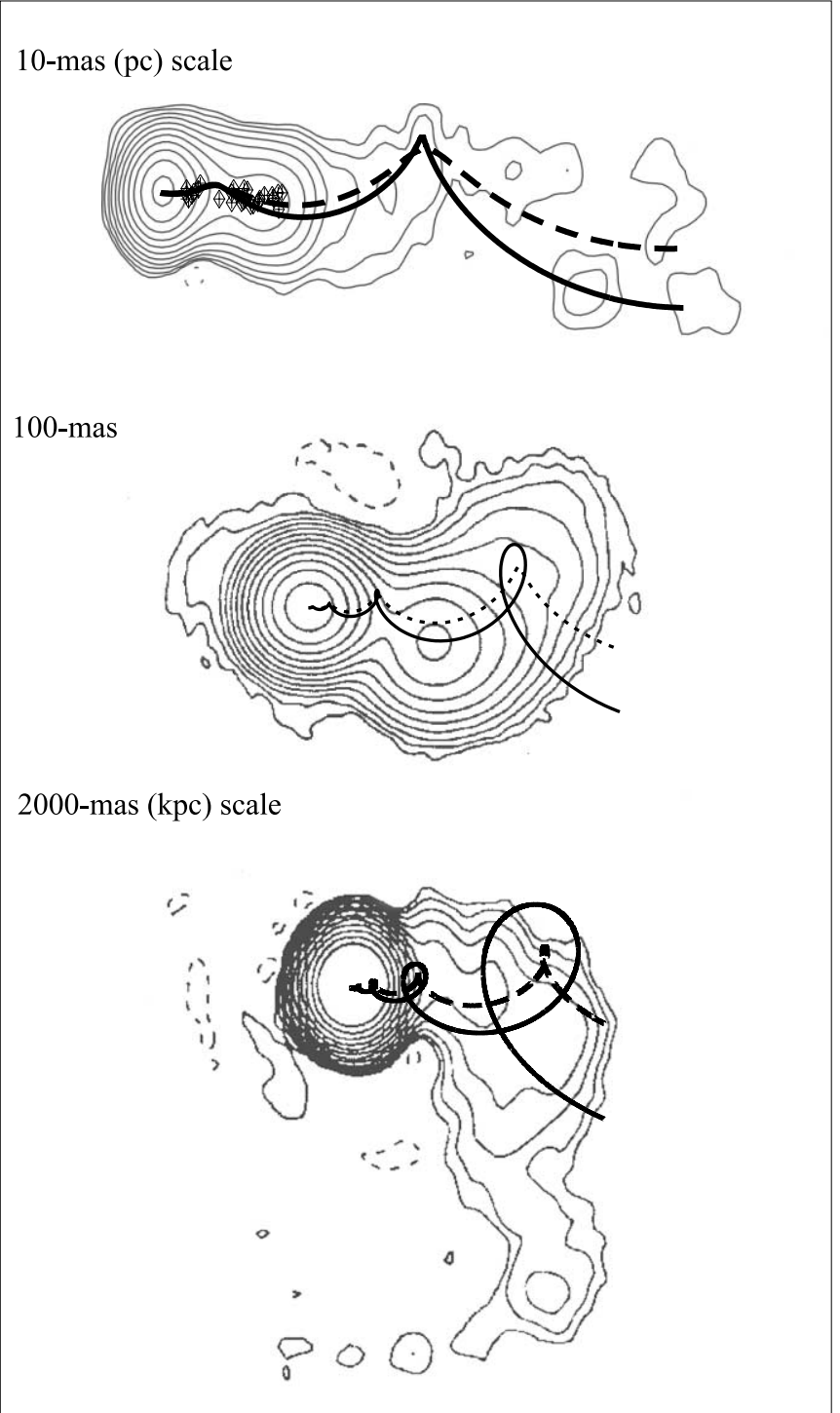

FIG. 6.- Helical curves superposed onto VLBI maps. The helical curves are the same as in Fig. 5. Top: 10 mas map of Kellermann et al. (1998). Middle: 100 mas scale map of Britzen et al. (1999). Bottom: 2000 mas scale map of Britzen et al. (1999).

The dashed curve shows an alternative fitting to our data with small changes in the helical parameter. The ratio of the amplitude of the helix to the jet radius $\xi$ and the amplitude growth $d_{1}$ were made slightly smaller with $\xi=0.9$ and $d_{1}=1.1$. This gives $R_{1}=0.059$ mas and $\Psi=0.44$, with all other parameters remaining unchanged. Also, the conical helix was rotated by 1.5 counterclockwise. All of this resulted in a better fit to our data (parsec scale) while still being satisfactory for helical features present in the 100 mas scale map; however, the largest change of helical trajectory between the two models appears at kiloparsec scale.

\subsection{Large-Scale Structure}

An interesting question that became evident was whether the structures present on the radio maps at kiloparsec scale would be associated with helical structures. In particular, orthogonal misalignment between the orientation of the jets in the parsec and kiloparsec scales can be explained in terms 
of the geometrical saturating helical model by Conway \& Murphy (1993), with the parsec scale being the result of emission from superluminal components moving along the surface of the cone and the kiloparsec scale being the result of emission from saturated helical components moving parallel to the cone axis. In the case of $1803+784$, the orthogonal misalignment is physically bent, and the entire helical jet could be taken as a single jet of $6 \mathrm{kpc}$ in extension and perpendicular to helical jet as a dogleg structure forming the outer jet of $1803+784$. We will discuss this morphology as generated by a straight cone axis and by a curved cone axis.

\subsection{Straight Cone Axis}

We consider a simple helical jet without bending the cone axis. The radio structures at 10 and 100 mas are well described by the helical curve, represented by the solid line in Figure 5 (top and middle panels). The helical structures at kiloparsec scale are shown on the bottom panel of Figure 5. The radio map is shown on the bottom of Figure 6. The outermost loop shows an arc, a north-south path on the eastern side of the last loop that may correspond to a radio structure shown between 500 and -500 mas in declination and between -600 and -1000 mas in right ascension on the 2000 mas scale map of Britzen et al. (1999). At the end of this trajectory, about 1000 mas (toward the south), the jet components may decouple from the conical jet and head toward the south. The radio structure on the western side of 2000 mas scale map suggests a superposition of helical trajectories. The radio structure in this region could be the result of emission produced from jet components moving along a helical trajectory between -700 and -1100 mas (parallel to the right ascension axis) and ending northwest at about $(-1200,200)$. However, the agreement between helical trajectories and the structure in the radio map ends here. While radio structures at kiloparsec scale seem to have their origin in helical motion, and even orthogonal structures seem to be related to helical structures especially near the cone axis, a simple helical morphology would not be able to reproduce the well-defined elongated structure extending farther south by several arcseconds. In fact, very high dynamic range observations from the VLA (Antonucci et al. 1986) and Westerbork (Strom \& Biermann 1991) show a one-sided weak bridge extending south of the core and ending in a compact hot spot at $45^{\prime \prime}$ from the core.

A dogleg morphology could be attributed to collision between the jet and a massive cloud (Stocke et al. 1985). Because of collisional heating, an expansion of the deflected jet is expected. In the case of $1803+784$, the size of the secondary component $\left(45^{\prime \prime}\right)$ and the distance from the core do not show an indication of expanded structure, suggesting an almost purely elastic collision. However, a helical motion would provide a favorable configuration for producing orthogonal structure. If there were a small deflection of the jet, just to straighten it, and the jet components continued to move along, for a distance comparable to fraction of a $\mathrm{Mpc}$, the north-south component would have a size of about 270 $\mathrm{kpc}$ (on the plane) and end in a compact hot spot. Recent hydrodynamic simulations by Higgins, O’Brien, \& Dunlop (1999) have shown that a variety of structures may have been produced from simple jet-cloud collisions. It appears that moderate Mach numbers and density contrasts are needed to produce bends in the radio structure that may be appropriate for helical components at distances as large as 6 $\mathrm{kpc}$ from the core.

\subsection{Curved Cone Axis}

An alternative scenario can be described by a curved cone axis. We may have a physically curved cone axis as if it has been rotated counterclockwise in the parsec and the intermediate scales (as shown by dashed lines on top and middle panel of Fig. 5) and then rotate the cone axis clockwise with stronger curving (toward the south) of the jet cone axis as it approaches the kiloparsec scale. At this point, the saturated helical distortions would lie approximately parallel to the cone axis and proceed along the jet until the hot spot is produced at $45^{\prime \prime}$ from the core.

\subsection{Lifetime of Component $\mathrm{C} 2$}

Our study suggests that the VLBI components move along a helically curved jet. The superluminal components appear first as a stationary component at 0.5 mas. They reappear as superluminal components at about 1 mas from the core. In Figure 2 component $\mathrm{C} 2$ can be followed moving from 0.9 to 1.6 mas from the core, corresponding to a duration of $11 \mathrm{yr}$. This is one of the longest observed timescales for superluminal components. Blazars such as BL Lac itself (Tateyama et al. 1998), OJ 287 (Tateyama et al. 1999), or even quasar 3C 279 (Wehrle et al. 2001) show a lifetime of about 2 or $3 \mathrm{yr}$. The observed timescale is related to the proper timescale (Qian et al. 1996) by $\Delta t_{\text {obs }}$ $=\Delta t_{\text {proper }}(1+z) / \delta \gamma$, where $\gamma$ is the Lorentz factor of the bulk relativistic motion and $\delta$ is the Doppler factor. Let us first assume that the proper timescale and Lorentz-Doppler factor may self-regulate in such a way that the observed timescales from BL Lac objects as well as quasars would converge to an observed timescale of about 2 or $3 \mathrm{yr}$. Clearly enough, such an assumption does not hold for $1803+784$, indicating either an intrinsic long duration of the component or an unlikely lower than typical Lorentz-Doppler factor for a given object. Because we have no direct information about the intrinsic duration of components, we may examine whether jet kinematics of $1803+784$ would be compatible with a Lorentz-Doppler factor lower than that expected for a BL Lac object. The Doppler factor of $1803+784$ derived from variability and synchrotron selfCompton arguments gives $\delta \simeq 7$ (Lähteenmäki \& Valtaoja 1999). The Lorentz factor can be obtained using $\gamma=\left(\beta_{\mathrm{app}}^{2}+\delta^{2}+1\right) / 2 \delta \simeq 4$, with $\beta_{\mathrm{app}}=2 c$. This is also in good agreement with the Lorentz factor estimated using the "viewing angle" of the jet, $\gamma=1 / \sin \left(11^{\circ}\right) \sim 5.2$. All this gives a rather high Lorentz-Doppler factor for a BL Lac object, implying that the duration of component $\mathrm{C} 2$ is intrinsically long. The quasar 3C 279 is an example of a source exhibiting short and long observed timescales in a single source. In addition to the superluminal components of short timescales of $3 \mathrm{yr}$, one of the superluminal components has had a lifetime of $6 \mathrm{yr}$ and shows no sign of fading (Wehrle et al. 2001).

\section{CONCLUSIONS}

We have presented 11 VLBI maps derived from geodetic VLBI observations of $1803+784$. The source presents a core-jet structure with expansion occurring almost entirely westward on the axis of right ascension. The jet component 
shows an elongated structure that is composed of multiple, closely spaced components. Three superluminal components were identified in our maps for the period between 1988 and 1999. The expansion rate of superluminal compo-

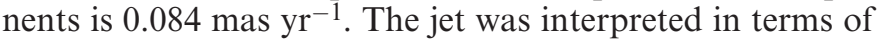
helical structure. At this point, published VLBI maps from Kellermann et al. (1998) and Britzen et al. (1999), showing helical structures from parsec to kiloparsec scale, enabled us to build a well-constrained helical model with a jet having a half-opening angle of 0.4 , pitch angle of 2.36 and an angle of cone axis to the line of sight of $11^{\circ}$. The morphology of $1803+784$ could be described by a helical jet with a size of about $6 \mathrm{kpc}$ (a deprojected size would be about $30 \mathrm{kpc}$ ) and a much larger north-south component perpendicular to the helical jet (cone axis), which would have a size of about 270 $\mathrm{kpc}$ (on the plane).
C. E. T. acknowledges the grant received from FAPESPFundação de Amparo a Pesquisa do Estado de São Paulo (proc. n. 96/6267-1) to undertake 3 months of work with geodetic VLBI data at USNO (US Naval Observatory). The referees's suggestions were of great value in improving the content of this paper. We thank A. V. R. Silva for reading the paper and V.S. Pereira for helping us with Figure 6. The University of Michigan Radio Astronomy Observatory is supported by the National Science Foundation and by funds from the University of Michigan. The Fortaleza VLBI facility was built and is operated with partial support from US NOAA, NASA/Goddard, USNO, Brazil ministry of Science and Technology, MCT-FINEP, and CRAAE (joint center between USP, Mackenzie, UNICAMP universities, and INPE, Brazil).
Antonucci, R. R. J., Hickson, P., Olsezewski, E. W., \& Miller, J. S. 1986, AJ, 92, 1

Biermann, P., et al. 1981, ApJ, 247, L53

Britzen, S., \& Krichbaum, T. P. 1995, in Proc. 10th Working Meeting on European VLBI for Geodesy and Astrometry, ed. R. Lanotte \& G. Bianco (Matera: ASI), 172

Britzen, S., Witzel, A., Krichbaum, T. P., \& Muxlow, T. W. B. 1999, NewA Rev., 43, 751

Carter, W. E., Robertson, D. S., \& MacKay, J. R. 1985, J. Geophys. Res., 90,4577

Charlot, P. 1990, A\&A, 229, 51

Coates, R. J., Frey, H., Mead, G. D., \& Bosworth, J. M. 1985, IEEE Trans. Geosci. \& Remote Sensing, GE-23, 360

Conway, J. E., \& Murphy, D. W. 1993, ApJ, 411, 89

Eubanks, T. M., et al. 1991, Earth Orientation...Program (IERS Tech. Note 8; Paris: IERS), 73

Fey, A. F., Clegg, A. W., \& Fomalont, E. B. 1996, ApJS, 105, 299

Gabuzda, D. C. 2000, in Astrophysical Phenomena Revealed by Space VLBI, ed. H. Hirabayashi, P. G. Edwards, \& D. W. Murphy (Sagamihara: Inst. Space \& Astronautical Sci.), 121

Gabuzda, D. C., \& Cawthorne, T. V. 1996, MNRAS, 283, 759

Gabuzda, D. C., Cawthorne, T. V., Roberts, D. H., \& Wardle, J. F. C 1992, ApJ, 388, 40

Gabuzda, D. C., Mullan, C. M., Cawthorne, T. V., Wardle, J. F. C., \& Roberts, D. H. 1994, ApJ, 435, 140

Ghisellini, G., Padovani, P., Celotti, A., \& Maraschi, L. 1993, ApJ, 407, 65

Hardee, P. E. 1987, ApJ, 318, 78

Higgins, S. W. O'Brien, T. J., \& Dunlop, J. S. 1999, MNRAS, 309, 273

Kellermann, K. I., Vermeulen, R. C., Zensus, J. A., \& Cohen, M. H. 1998, AJ, 115, 1295

Kollgaard, R. I., Wardle, J. F. C., Roberts, D. H., \& Gabuzda, D. C. 1992, AJ, 104,1687
Krichbaum, T. P., Witzel, A., Graham, D. A., Schalinski, C. J., \& Zensus, J. A. 1993, in Subarcsecond Radio Astronomy, ed. R. J. Davies \& R. S. Booth (Cambridge: Cambridge Univ. Press), 260

Lähteenmäki, A., \& Valtaoja, E. 1999, ApJ, 521, 493

Lawrence, C. R., Zucker, J. R., Readhead, A. C. S., Unwin, S. C., Pearson, T. J. \& Xu, W. 1996, ApJS, 107, 541

Pearson, T. J., \& Readhead, A. C. S. 1984, ARA\&A, 22, 97

. 1988, ApJ, 328, 114

Qian, S. J., Krichbaum, T. P., Zensus, J. A., Steffen, W., \& Witzel, A. 1996, A\&A, 308, 395

Rogers, A. E. E., et al. 1983, Science, 219, 51

Ros, E., Marcaide, J. M., Guirado, J. C., Ratner, M. I., Shapiro, I. I., Krichbaum, T. P., Witzel, A., \& Preston, R. A. 1999, A\&A, 348, 381

Smith, D. E., \& Turcotte, D. L., eds. 1993, Geodynamics Ser. 23 , Contributions of Space Geodesy to Geodynamics: Crustal Dynamics (Washington: AGU)

Stocke, J. T., Burns, J. O., \& Christiansen, W. A. 1985, ApJ, 299, 799

Strom, R. G., \& Biermann, P. L. 1991, A\&A, 242, 313

Tateyama, C. E., Kingham, K. A., Kaufmann, P., Piner, B. G., Botti, L. C. L., \& de Lucena, A. M. P. 1999, ApJ, 520, 627

Tateyama, C. E., Kingham, K. A., Kaufmann, P., Piner, B. G., de Lucena, A. M. P., \& Botti, L. C. L. 1998, ApJ, 500, 810

Valtaoja, E., Lähteenmäki, A., Teräsranta, H., \& Lainela, M. 1999, ApJS, 120,95

Vicente, L., Charlot, P. \& Sol, H. 1996, A\&A, 312, 727

Wagner, S. J., \& Witzel, A. 1995, ARA\&A, 33, 163

Wehrle, A. E., Piner, B. G., Unwin, S. C., Zook, A. C., Xu, W., Marscher, A. P., Teräsranta, \& Valtaoja, E. 2001, ApJS, 133, 297

Witzel, A Schalinski, C. J., Johnston, K. J., Biermann, P. L., Krichbaum, T. P., Hummel, C. A., \& Eckart, A. 1988, A\&A, 206, 245 